\title{
Drag reduction in a class 8 truck - scaled down model
}

\author{
Vishwa Krishna. $\mathrm{R}^{1}$, Suwathy. $\mathrm{R}^{2}$, Pragadeesh. $\mathrm{M}^{1}$, and Venkatesan. $\mathrm{M}^{1, *}$ \\ ${ }^{1}$ School of Mechanical Engineering, SASTRA Deemed University, Tirumalaisamudram, Thanjavur - 613401, Tamilnadu, India \\ 2 Department of Aerospace Engineering, Madras Institute of Technology, Chennai-600044, Tamilnadu, India
}

\begin{abstract}
Trucks are heavy load vehicles used mainly for commercial transport operations. There are several classes of heavy duty commercial vehicles classified based on the weight loaded. More than $50 \%$ of the engine output power in such trucks is utilized to overcome the drag. Drag force in automobiles is the resistance offered by air on vehicles at higher speeds. Class 8 trucks suffer higher drag when compared to other classes. In the present work, a numerical model is developed using a commercial code ANSYS FLUENT to predict the drag coefficient value. The effects of gap width and cab front radius with a constant fairing is analysed using the numerical model developed. A Class 8 model truck with minimal drag coefficient having constant fairing and optimized gap width between the trailer and cab is proposed.
\end{abstract}

\section{Introduction}

Heavy trucks which contain a cab and trailer come across more drag than conventional vehicles because of the vortex formation in the gap. The conventionally used class 8 truck is found to have bluffed and unstreamlined profile by design. This profile results in increased drag due to flow separation phenomenon. Many research works were reported on aerodynamic design of heavy commercial vehicles to reduce the resistance force and increase the engine efficiency in a feasible way. The drag force can be minimized by fairing the shape or implementing drag reducing devices. Also with proper design of geometry of the front cab of a given truck, the drag coefficient can be optimized. Such optimized geometries will result in decreased fuel consumption.

Allan [1] studied the effect of drag experimentally in a tractor trailer model. The cab front edge was rounded and the effect of it on drag was studied. Such experimental methods cannot be implemented for all possible design variations or such an implementation is not economical. With increase in computational resources, employing computational techniques is an easier solution in such cases where an optimized profile can be found out numerically. To verify the results, limited experiments can be done. Osth and Krajnovic [2] used large eddy simulation and studied the flow through the tractor trailer model numerically for four different cases. The gap width was changed and the cab front edge was rounded in and the effect was studied. Bettle et al. [3] investigated the speed of truck under the conditions of crosswind on a standard sized North American transport truck. Various aerodynamic parameters for different relative wind directions were varied and the parameter which would tend to overturn a truck was detailed. Hu et al. [4] made inferences from latter and numerically analysed the effect of aerodynamic loads with SST k-omega turbulence model in a heavy duty truck. The results showed a decrease in side force with the protection of fence and strengthening of rolling and yawing moments. Wang et al. [5] investigated the aerodynamic characteristics of three rear shapes numerically and experimentally in a wind tunnel to examine the effects

As a result of induced accidents in UK, Coleman \& Baker [6] studied the aerodynamic forces experienced by a scaled down articulated lorry in a wind tunnel experimentally. The side flow oscillations were largely caused by turbulence buffeting and lift force oscillations that were caused by wake vortex shedding. Pragadeesh et al. [7] numerically analysed eight different models with constant gap width. Among the eight cases, the addition of a fairing to a truck which has its cab rounded to optimal radius was observed to have minimal drag and eddies to a convincing level.

To reduce drag, proper fairing methods like rounding the sharp edges to a certain radius, and fixing fairing plates between cab and trailer can be adopted. In the present work, co-efficient of drag is found for nine different cases in scaled down model of a class 8 truck. Among these nine cases, three cases which have got minimal drag are analysed to have minimal drag. The observed drag values are more dependent on the vortex that is formed in the gap width. The vorticity magnitude for both z-direction are calculated individually and their effect on drag is detailed. The model with minimal drag value and its design cases are presented.

*orresponding author: mvenkat@mech.sastra.edu 


\section{Numerical model}

\subsection{Size of the domain}

The scaled down model of a class 8 truck is shown in Fig. 1. It consists of a cab and a trailer placed in a domain of $8000 \times 2104.5 \times 1708 \mathrm{~mm}$. The dimensions of the entire model is calculated based on rear box width $\mathrm{b}=0.305 \mathrm{~m}$. The dimensions of the cab are $204.35 \mathrm{x}$ $280.6 \times 280.6 \mathrm{~mm}$. The dimensions of the trailer are $762.5 \times 280.6 \times 305 \mathrm{~mm}$. Cab and trailer are connected by a cylinder of diameter $24.4 \mathrm{~mm}$. The gap width is varied in the present work. The height of cab and trailer above the ground are $64.05 \mathrm{~mm}$ and $152.5 \mathrm{~mm}$ respectively. Air with a velocity of $24.4 \mathrm{~m} / \mathrm{s}$ is supplied at the inlet of the domain. Absolute pressure condition is chosen for exit of the domain.

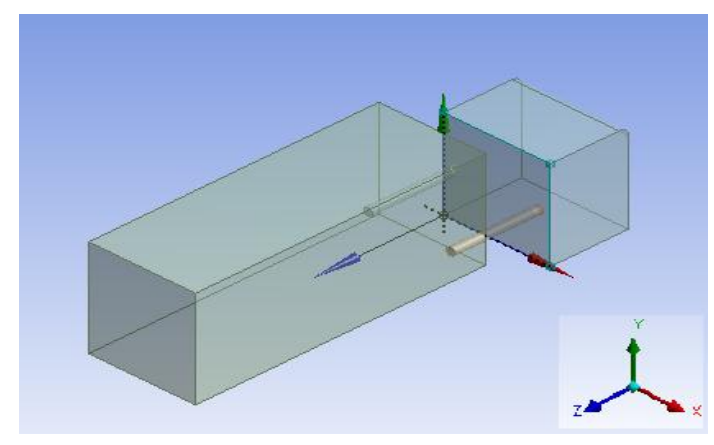

Fig. 1. Numerical model

\subsection{Governing equations}

The mass momentum equations along with standard $\mathrm{k}-\omega$ turbulence model are used to model the trailer using commercial Fluent code Ansys [8] and are shown in eqn. (1), (2) (3) and (4).

$$
\begin{gathered}
\nabla \cdot(v)=0 \\
\frac{\partial(\rho v)}{\partial t}+\nabla \cdot(\rho v v)=-\nabla p+\nabla \cdot(\tau)+\rho g \\
\frac{\partial}{\partial t}(\rho k)+\frac{\partial}{\partial x_{i}}\left(\rho k u_{i}\right)=\frac{\partial}{\partial x_{j}}\left(\Gamma_{k} \frac{\partial k}{\partial x_{j}}\right)+G_{k}-Y_{k}+S_{k} \\
\frac{\partial}{\partial t}(\rho \omega)+\frac{\partial}{\partial x_{i}}\left(\rho \omega u_{i}\right)=\frac{\partial}{\partial x_{j}}\left(\Gamma_{\omega} \frac{\partial \omega}{\partial x_{j}}\right)+G_{\omega}-Y_{\omega}+S_{\omega}
\end{gathered}
$$

The above equations are solved simultaneously to find velocity, pressure and turbulence variables.

The model is validated with air having its flow behaviour in turbulent regime. Hence, k- $\omega$ SST model is used in the estimation of coefficient of drag for the above model and are shown in eqns (5) \& (6). The choice of this model is done based upon the validation study in which other turbulences models used didn't predict the experimental results closely. Thed model merges the Wilcox $\mathrm{k}-\mathrm{w}$ model for near walls and standard $\mathrm{k}-\varepsilon$ model away from walls using a blending function. Eddy viscosity formulation is changed to account for the transport effects of principle turbulent shear stress. The coefficient of drag obtained is 1.025 .
This value obtained is in close agreement with Allan [1] which validates the present model developed. Grid independent study is done in such a way that the value of drag coefficient does not change with increase in number of grids and optimizing computational time. Based on this study 19, 48, 729 number of mesh elements is used. More details on the study are available in Pragadeesh et al. [7]. For pressure-velocity coupling, SIMPLE algorithm is used. Second order upwind momentum is used in spatial discretization.

\section{Results and discussions}

The study was carried out for nine models as shown in Table 1. Three models among these considered models showed minimal drag. So flow analysis of these three models is done with variations in gap width and modifications of the cab radius. Flow for rest of the models is not explained in this present study. It is found that vortex formation in the gap width resulted in increase in drag. If the vortex magnitude decreases, the drag value also decreases. Modification is done on these three models with addition of fairing and rounding the cab surface. The top surface of the plate is rounded to $600 \mathrm{~mm}$ for all the three models considered.

Table 1. Study for nine models

\begin{tabular}{|l|l|l|l|}
\hline S.No & $\begin{array}{l}\text { Radius of cab front } \\
\text { surface }(\mathrm{mm})\end{array}$ & $\begin{array}{l}\text { Gap width } \\
(\mathrm{mm})\end{array}$ & $\begin{array}{l}\text { Coefficient } \\
\text { of drag }\end{array}$ \\
\hline 1 & 0 & 110 & 0.945 \\
\hline 2 & 0 & 200 & 0.963 \\
\hline 3 & 0 & 230 & 0.968 \\
\hline 4 & 24.4 & 110 & 1.032 \\
\hline 5 & 24.4 & 200 & 1.008 \\
\hline 6 & 24.4 & 230 & 0.998 \\
\hline 7 & 48.8 & 110 & 0.995 \\
\hline 8 & 48.8 & 200 & 0.952 \\
\hline 9 & 48.8 & 230 & 0.925 \\
\hline
\end{tabular}

The models are kept in such a way that air flows in Zdirection and flow separates in $\mathrm{x}$-direction. Flow separation along Y-direction is minimised by rounding the cab radius and by addition of a plate (fairing) above the cab and trailer. Vorticity magnitude is measured along Z-direction. The velocity contours along Zdirection is obtained so as to understand the vortex phenomena in Z-direction. It is observed in all the three models that the velocity of flow decreases along Zdirection as air moves inwards due to flow separation. A larger vortex is formed in the gap width due to flow separation. The magnitude of vorticity is measured along Z-direction and a plot is drawn for all the three cases. Since the flow is in Z-direction, the vortex variation along Z-direction is plotted. The vorticity magnitude is defined as a pseudo vector field which is used for 
describing the vortex motion in a continuum in a specified area. Mathematically it can also be defined as the curl of flow field velocity vector given by (5).

$$
\vec{\omega}=\nabla \times \vec{V}
$$

\subsection{Model 1 - Rounded cab front surface with medium gap width}

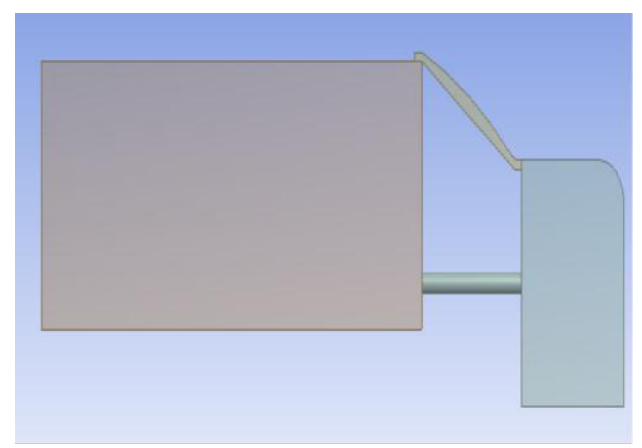

Fig. 2. Model 1

In this model, the front surface is rounded for the cab and is shown in Fig. 2. A medium gap width is maintained. The gap width is kept as $200 \mathrm{~mm}$ and the front surface of the cab is rounded to $48.8 \mathrm{~mm}$. Since cab is rounded, flow separation is reduced. Since the top surface of the fairing is curved, the velocity along the fairing is less. It is observed from the velocity contour that velocity near the cab front surface is small when compared to sharp edge as seen in Fig. 3. The vorticity magnitude is plotted along $\mathrm{z}$-direction in the gap width region and is shown in Fig. 4. The vorticity magnitude is low near the cab. Along Z-direction, the vorticity magnitude gradually increases till $Z=0.045 \mathrm{~m}$ due to gradual change in velocity. There are minor variations in vorticity magnitude due to lower velocity values till $\mathrm{Z}=$ $0.095 \mathrm{~m}$. After that due to larger velocity variations, vorticity magnitude reaches its maximum at the start of the trailer. Velocity contours and their respective plots are shown below. As a result of these flow variations, the average drag coefficient obtained is 0.952

\subsection{Model 2 - Sharp cab front surface with smaller gap width}

In the second model, the gap width is kept as $110 \mathrm{~mm}$ and front surface of the cab is kept sharp as shown in Fig. 5. A larger portion of air moving over the cab has higher velocity because of this sharper edge. The velocity near the fairing is minimal due to curved geometry of fairing. There is less amount of air near the cab due to which vorticity magnitude is also low. Along $\mathrm{Z}$-direction there is a steady increase in velocity due to which voriticity increases until $Z=0.025 \mathrm{~m}$. Since the

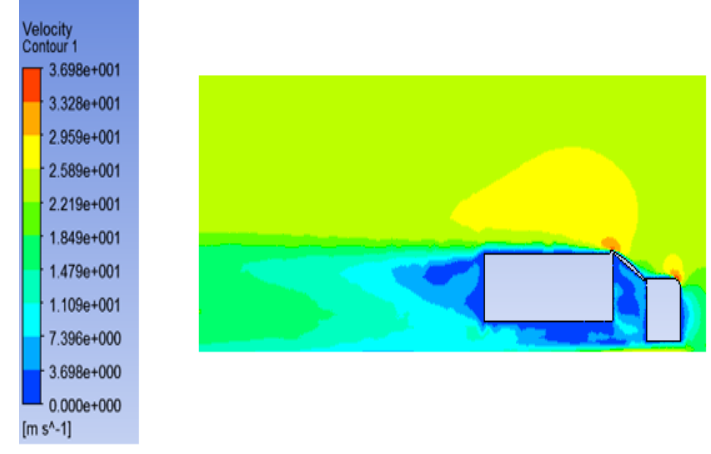

Fig. 3. Velocity contour for model 1

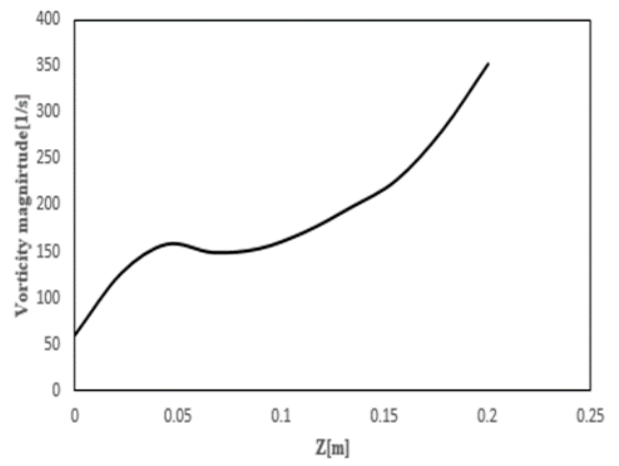

Fig. 4. Vorticity vs z-distance for Model 1

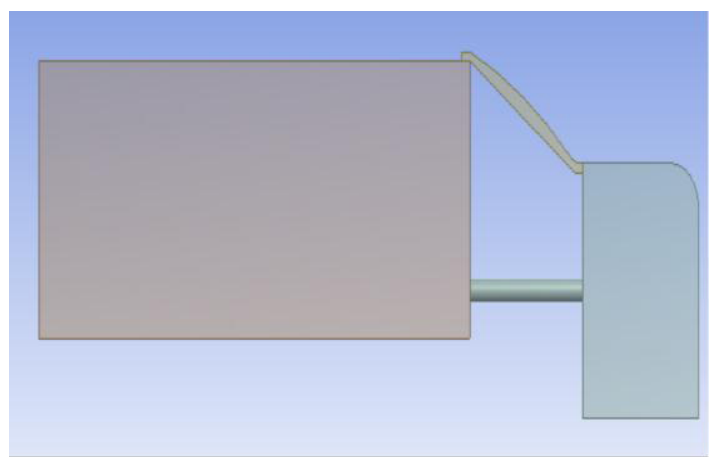

Fig.5. Model 2

amount of air after $Z=0.025 \mathrm{~m}$ decreases, the vorticity magnitude decreases till $Z=0.05 \mathrm{~m}$. After that, magnitude of vorticity increases till $\mathrm{Z}=0.09 \mathrm{~m}$ because of large variations in velocity. In the latter part of the gap width, voritcity magnitude starts decreasing because air will leave the gap width. The coefficient of drag obtained in model 2 is 0.945 . The velocity contour and plots of vorticity magnitude vs z-distance is shown respectively in Fig 6 \& Fig 7. 

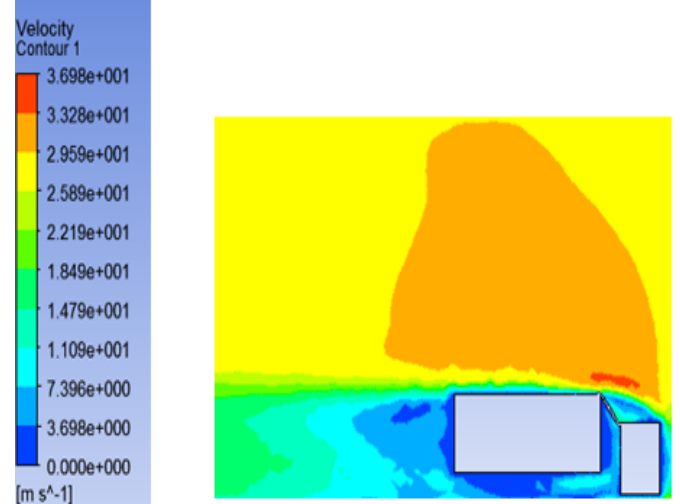

Fig.6. velocity contour for model 2

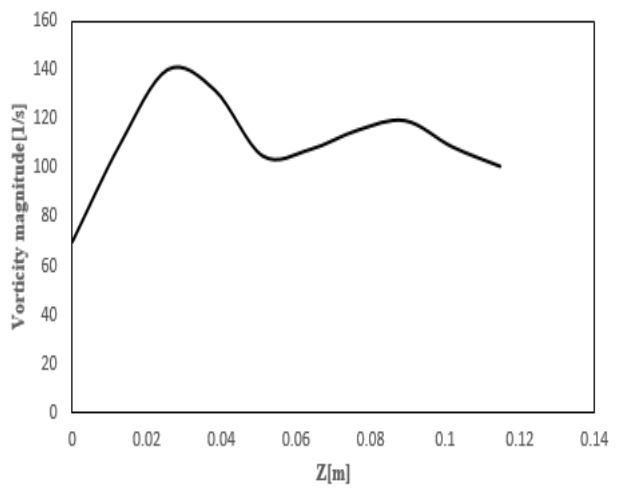

Fig. 7. vorticity vs z-distance for Model 2

\subsection{Model 3 - Rounded cab front surface with larger gap}

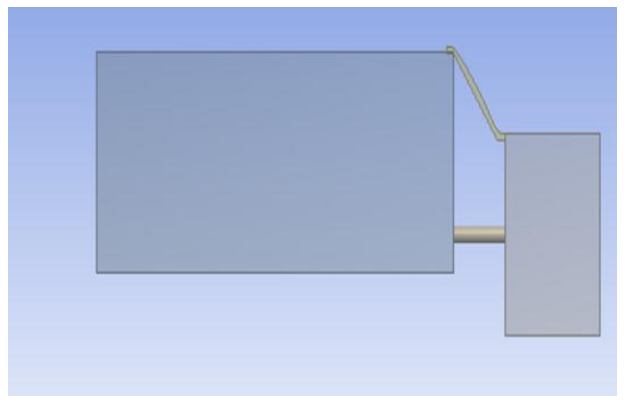

Fig. 8. Model 3

The model 3 shown in Fig. 8, has its front surface of the cab rounded to $48.8 \mathrm{~mm}$ and a gap width as $230 \mathrm{~mm}$. The velocity value is minimal at the front surface, rounded $\mathrm{cab}$ and curved top fairing. Similar to the previous models, vorticity magnitude is minimal near the cab. Along the Z-direction, there are multiple regions where the vorticity magnitude increases and decreases gradually. The region where vorticity has gradual increase are from $\mathrm{Z}=0-0.025 \mathrm{~m}, \mathrm{Z}=0.05-0.075 \mathrm{~m}$ and $\mathrm{Z}=0.1-0.175 \mathrm{~m}$. The region where vorticity has minimal variations are from $Z=0.025-0.05 \mathrm{~m}, Z=0.075-0.1 \mathrm{~m}$ and $\mathrm{Z}=0.175-0.2 \mathrm{~m}$. The region of gradual increase is due to increase in velocity. The region of minimal variations is due to low velocity. After $\mathrm{Z}=0.2 \mathrm{~m}$ there is steep increase due to rapid increase in velocity. These are evident from the contours and plots given shown in Fig. $9 \& 10$. The coefficient of drag obtained in this model is 0.925 . The velocity contours and vorticity magnitude are show in Fig. 9 \& 10.

It can be seen from the plot shown in Fig. 10, that vorticity magnitude is less at start of cab and trailer near the origin. Along the Z-direction the magnitude of vorticity increases than the value which was measured at the start of trailer.

From the above three models it can be observed that, the effect of gap width, additional fairing and rounding the cab front surface has its impact on formation of vortex in the gap width. It is very well clear that the vorticity magnitude near the cab is less than the vorticity magnitude near the trailer. For a truck having smaller gap width, the vorticity magnitude drop and raise. For a truck having larger gap width, the vorticity magnitude did not show major variations. But the velocity showed minimal variations at certain locations due to which vorticity magnitude is maintained constant. The vorticity magnitude showed variations at certain locations due to gradual changes in velocity.
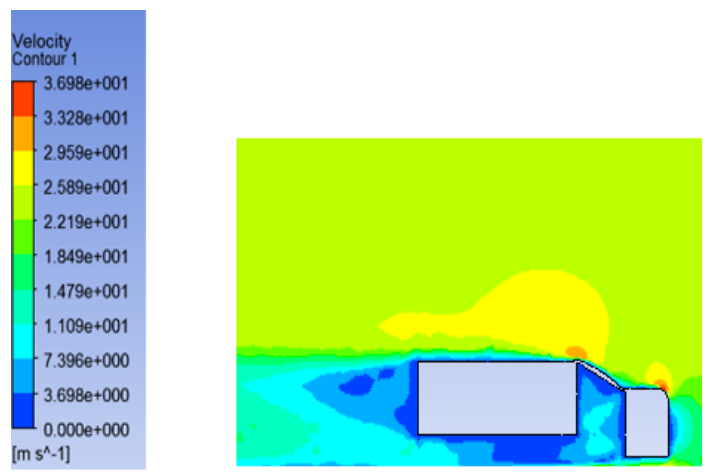

Fig.9. velocity contour for model 3

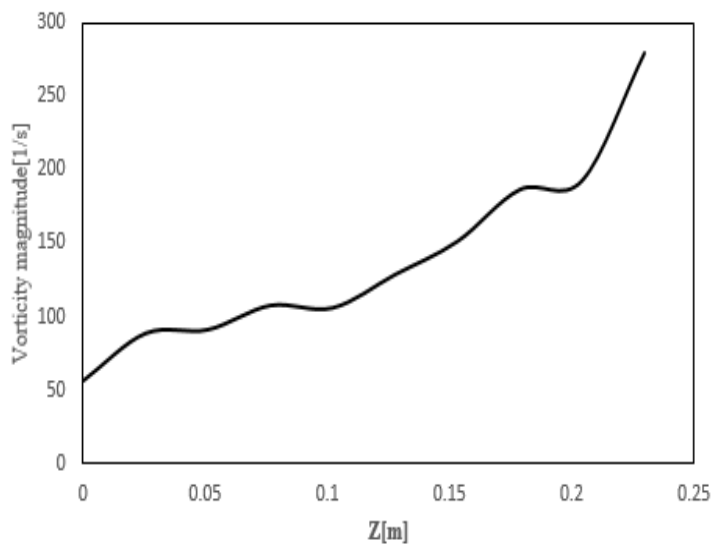

Fig.10. vorticity vs z-distance for Model 3

\section{Conclusions}

In the present study, a numerical model is developed using a commercial code ANSYS FLUENT to predict the drag coefficient value of class 8 trucks. The results predicted are compared with experimental results 
available in literature for validation. The effects of gap width and cab front radius along with a constant fairing are analysed using the validated numerical model. It was observed from the analysis of three models, that minimal drag coefficient for a given truck depended upon the gap width and the magnitude of vortex. Vortex formation in the gap played an important role in drag reduction. Third model which a rounded cab front surface with larger gap width had resulted in minimal drag coefficient.

\section{References}

1. J.W. Allan, Journal of Wind Engineering and Industrial Aerodynamics, no. 9 (1-2): 125-136 (1981).

2. J. Östh, and S. Krajnović, Journal of Wind Engineering and Industrial Aerodynamics 102: 3647(2012).

3. J. Bettle, A. G. L. Holloway, and J. E. S. Venart, Journal of Wind Engineering and Industrial Aerodynamics 91, no. 5: 573-592 (2003).

4. H. Xing-jun, Q. I. N. Peng, L. I. A. O. Lei, G. U. O. Peng, Jing-yu WANG, and Y. A. N. G. Bo, Journal of Hydrodynamics, Ser. B 26, no. 3: 394-399 (2014).

5. Y. Wang, Y. Xin, Zh Gu, Sh Wang, Y. Deng, and X. Yang, Journal of Applied Fluid Mechanics 7, no. 4 (2014).

6. S.A. Coleman, and C. J. Baker, Journal of Wind Engineering and Industrial Aerodynamics 53, no. 3: 401-429 (1994).

7. M. Pragadeesh, R. Suwathy, M. Venkatesan, In International conference on design and manufacturing (IIITDM Kancheepuram December 2016), (Paper No.64).

8. ANSYS FLUENT Theory Guide: ANSYS. Inc. (2013). 